\title{
Manejo multimodal para reabilitação oral de paciente irradiado em cabeça e pescoço:
}

$$
\text { relato de caso }
$$

Multimodal approach for oral rehabilitation in an irradiated head and neck patient: a case report

Manejo multimodal para la rehabilitación oral de un paciente irradiado en la cabeza y el cuello: reporte de un caso

Recebido: 29/04/2020 | Revisado: 29/04/2020 | Aceito: 01/05/2020 | Publicado: 07/05/2020

Natália Silva Andrade

ORCID: https://orcid.org/0000-0001-5945-8401

Universidade Federal de Sergipe, Brasil

E-mail: natalia.andrade@ufs.br

Adriana Ferreira Granchelli

ORCID: https://orcid.org/0000-0001-9123-378X

Universidade de São Paulo, Brasil

E-mail: afgcd@uol.com.br

Marina Gallottini

ORCID: https://orcid.org/0000-0001-6071-5110

Universidade de São Paulo, Brasil

E-mail: mhcgmaga@usp.br

Luana Campos

ORCID: https://orcid.org/0000-0002-2747-2812

Universidade de Santo Amaro, Brasil

E-mail: lucampos@prof.unisa.br

Ricardo Yudi Tateno

ORCID: https://orcid.org/0000-0001-8678-689X

Universidade de Santo Amaro, Brasil

E-mail: ricardotateno@gmail.com

Luiz Felipe Palma

ORCID: https://orcid.org/0000-0002-4472-6941 
Universidade Federal de São Paulo e Universidade Ibirapuera, Brasil

E-mail: luizfelipep@hotmail.com

Fabiana Martins

ORCID: https://orcid.org/0000-0002-4352-7959

Universidade de São Paulo, Brasil

E-mail: fmoliveira@prof.unisa.br

\title{
Resumo
}

O objetivo do presente estudo foi relatar um caso clínico de abordagem multimodal para a reabilitação oral de paciente do sexo masculino, 69 anos, irradiado em cabeça e pescoço (radioterapia conformacional 3D, dose total de radiação de 70Gy), após metástase em laringe de câncer de intestino. Na consulta inicial, o paciente queixava-se de ausências dentárias e sensação de boca seca. Além do mais, foram observadas lesões de cárie de radiação na maior parte dos dentes, os quais também exibiam grande destruição coronária. $\mathrm{O}$ plano de tratamento consistiu na exodontia do dente 27 com auxílio de terapia fotodinâmica antimicrobiana para cicatrização do alvéolo, seguida da realização de tratamento endodôntico com subsequente sepultamento de raízes residuais, restaurações e reabilitação com próteses parciais removíveis. O manejo odontológico de pacientes pós-irradiados em cabeça e pescoço deve ser cuidadosamente planejado e conduzido por uma equipe odontológica interdisciplinar, considerando as sérias consequências resultantes dos efeitos deletérios da radioterapia na cavidade oral. Desta forma, pode-se mitigar o impacto negativo da radioterapia na qualidade de vida destes pacientes.

Palavras-chave: Radioterapia; Tratamento odontológico; Neoplasias de cabeça e pescoço.

\begin{abstract}
The present study aimed to report a clinical case of a multimodal approach for the oral rehabilitation of a 69-year-old male patient who received radiotherapy in the head and neck (3D-conformal, total radiation dose 70Gy) for larynx metastasis from bowel cancer. At the first dental appointment, the patient complained of missing teeth and dry mouth. In addition, radiation caries lesions were observed in most teeth, which also exhibited extensive coronary destruction. The treatment planning consisted of oral environment adequacy by non-surgical periodontal therapy (tooth scaling and root planning) and extraction of tooth \#27 with the aid of antimicrobial photodynamic therapy for healing improvement. Following, endodontic treatment with subsequent decoronated of unrestorable roots and crown restorations were performed, as well as rehabilitation with removable partial dentures later. The
\end{abstract}


dental management of post-irradiated patients in the head and neck must be carefully planned and conducted by an interdisciplinary dental team, considering the serious consequences resulting from the deleterious effects of radiotherapy in the oral cavity. As such, the negative impact of radiotherapy on the patients' quality of life may be mitigated.

Keywords: Radiotherapy; Dental treatment; Head and neck neoplasms.

\section{Resumen}

El objetivo del presente estudio fue informar un caso clínico de un enfoque multimodal para la rehabilitación oral de un paciente masculino de 69 años de edad, irradiado en la cabeza y el cuello (radioterapia conformacional 3D, dosis total de 70Gy), después de metástasis en la laringe del cáncer de intestino. En la consulta inicial, el paciente se quejó de dientes perdidos y boca seca. Además, se observaron lesiones de caries por radiación en la mayoría de los dientes, que también exhibieron una gran destrucción coronaria. El plan de tratamiento consistió en la extracción del diente 27 con la ayuda de una terapia fotodinámica antimicrobiana para curar el alvéolo, seguido de un tratamiento endodóntico con posterior enterramiento de las raíces residuales, restauraciones y rehabilitación con prótesis parciales removibles. El tratamiento dental de los pacientes pos irradiados en la cabeza y el cuello debe ser cuidadosamente planificado y realizado por un equipo dental interdisciplinario, considerando las graves consecuencias que resultan de los efectos nocivos de la radioterapia en la cavidad oral. De esta forma, se puede mitigar el impacto negativo de la radioterapia en la calidad de vida de estos pacientes.

Palabras clave: Radioterapia; Tratamiento dental; Neoplasias de cabeza y cuello.

\section{Introdução}

O tratamento do câncer de cabeça e pescoço é baseado essencialmente em três modalidades terapêuticas que podem ser combinadas ou não, de acordo com a localização e o estágio da doença: radioterapia (RT), quimioterapia e cirurgia (Comodo et al., 2020; Palma et al., 2017, 2018, 2020). Nos últimos anos, com os avanços tecnológicos no campo da radiação oncológica, as técnicas bidimensionais foram gradativamente substituídas pelas tridimensionais, estas baseadas em imagens de tomografia computadorizada, proporcionando melhor controle das doses recebidas, melhores taxas de sobrevida do paciente e redução da incidência e severidade dos efeitos adversos da RT (Marta et al., 2014; Ray-Chaudhuri et al., 2013). Entretanto, tecidos não neoplásicos localizados dentro ou próximos aos campos de irradiação acabam inevitavelmente sendo atingidos, resultando em uma série de efeitos indesejáveis que podem ocorrer durante ou logo após o curso da RT e perdurar por toda a vida 
do paciente (Gonnelli et al., 2016a, 2016b).

A inexistência de técnicas que eliminem completamente esses efeitos levam a severas complicações, especialmente na cavidade oral, como mucosite, xerostomia, infeções orais, disgeusia, trismo e osteorradionecrose (Gupta et al., 2015; Kielbassa et al., 2006). Outra consequência grave da RT inclui o desenvolvimento de lesões de cáries de radiação, condição resultante de diversas alterações orais que levam a uma maior susceptibilidade à desmineralização dos tecidos duros dentais (Gupta et al., 2015).

Apesar da cárie de radiação ter etiologia multifatorial, a hipossalivação é considerada a principal causa (Kielbassa et al., 2006). Assim, tais lesões cariosas podem ser evitadas ou reduzidas quando as glândulas salivares são poupadas no planejamento da RT ou por meio de condutas odontológicas preventivas antes, durante e após o tratamento oncológico. Entretanto, se presentes, o manejo dessas lesões de cárie pode ser um desafio para o cirurgião-dentista (Gupta et al., 2015).

O objetivo do tratamento odontológico no paciente oncológico é preservar a dentição e as funções orais básicas. As estratégias de reabilitação devem ser interdisciplinares e multimodais, abrangendo medidas de prevenção, periodontia, dentística, endodontia, cirurgia e prótese (Kielbassa et al., 2006). Na presença de focos de infecção na cavidade oral, o profissional deve avaliar o risco de desenvolvimento de osteorradionecrose dos maxilares, uma das consequências mais severas da RT na região da cabeça e pescoço. Esta apresenta elevada morbidade e seu tratamento é desafiador, variando desde condutas extremamente conservadoras, como a proservação, até ressecções cirúrgicas radicais mutiladoras (RayChaudhuri et al., 2013).

$\mathrm{Na}$ luz destes fatos, o objetivo do presente estudo é relatar um caso clínico de abordagem multimodal para a reabilitação oral de paciente pós-radioterapia na região de cabeça e pescoço.

\section{Relato de Caso}

Paciente do sexo masculino, 69 anos de idade, leucoderma, procurou atendimento odontológico no Centro de Atendimento a Pacientes Especiais da Faculdade de Odontologia da Universidade de São Paulo (CAPE-FOUSP) relatando dificuldades em mastigar e comprometimento estético. Durante a anamnese, foi levantada história médica de adenocarcinoma em intestino tratado com cirurgia e quimioterapia, seguido de metástase em laringe (carcinoma epidermóide bem diferenciado microinvasivo em pregas vocais) que 
(CC BY 4.0) | ISSN 2525-3409 | DOI: http://dx.doi.org/10.33448/rsd-v9i7.4062

exigiu nova intervenção cirúrgica e RT conformacional tridimensional com dose total de 70Gy e finalizada há 7 anos. O paciente relatou ausência de quaisquer outras alterações sistêmicas, uso de medicações e histórico de etilismo crônico. Por outro lado, o tabagismo esteve presente por 37 anos, com abandono deste hábito há 12 anos.

$\mathrm{Na}$ história odontológica, a última visita do paciente ao dentista tinha sido há cinco anos, por fobia ao tratamento. O paciente não fazia uso de próteses dentárias e evidenciou sensação de boca seca e sangramento gengival durante a escovação.

Ao exame clínico, observou-se a presença de várias raízes residuais, dentes com destruição coronária, lesões de cárie ativa, cálculo dentário e gengivite (Figura 1 A e B). Ao exame radiográfico, verificou-se a presença de algumas raízes residuais com tratamento endodôntico realizado e outras não, além de cáries proximais (Figura $1 \mathrm{C}$ ). Assim, traçou-se um plano de tratamento odontológico multimodal, englobando diversas especialidades.

Para adequação do meio, foram inicialmente realizados raspagem, alisamento e polimento coronorradicular em todos os dentes/raízes residuais. Além do mais, a raiz residual do dente 27 foi extraída atraumaticamente e terapia antibiótica pós-operatória foi prescrita (amoxicilina 500mg de 8/8 horas, durante 7 dias). Foi utilizada também sessão única de terapia fotodinâmica antibacteriana com o corante fotossensibilizador azul de metileno à $2 \%$ agindo por 3 minutos, seguido da irradiação local com laser de baixa potência (MMOptics®, São Carlos, Brasil) no comprimento de onda de $660 \mathrm{~nm}$, potência de $40 \mathrm{~mW}$ e energia total de $4,8 \mathrm{~J}$, totalizando 2 minutos em um ponto central na área da ferida cirúrgica.

Seguiu-se com tratamento endodôntico do dente 35 e subsequente sepultamento radicular. Da mesma forma, sepultamentos radiculares de outras raízes residuais (dentes 11, $12,13,14,21,22,23,34,44,45)$ também foram realizados (Figura $1 \mathrm{C}$ ). 
Research, Society and Development, v. 9, n. 7, e192974062, 2020

(CC BY 4.0) | ISSN 2525-3409 | DOI: http://dx.doi.org/10.33448/rsd-v9i7.4062

Figura 1: Exame clínico e radiográfico inicial. A)Maxila. B)Mandíbula. C)Radiografia Panorâmica.

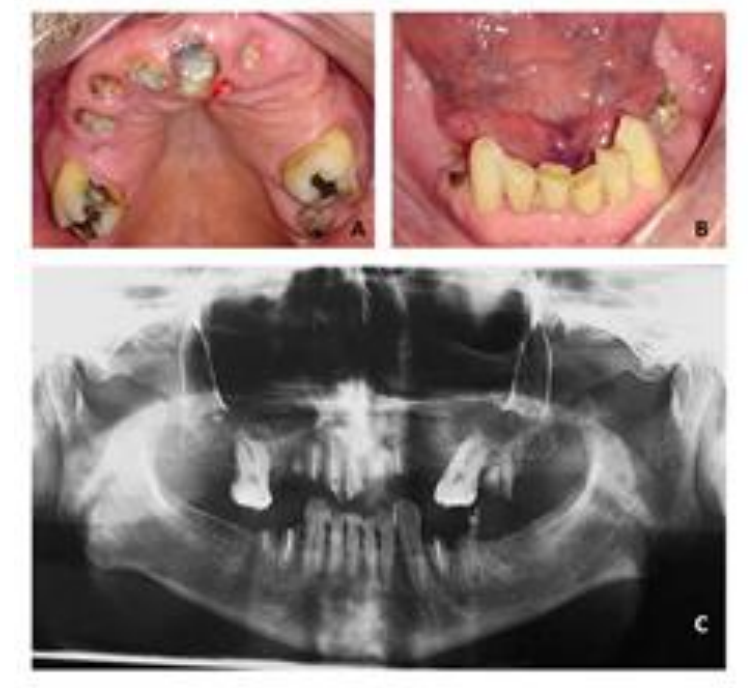

Fonte: Os Autores.

O dente 26 recebeu restauração de amálgama e os dentes 33 ao 43 receberam restaurações diretas em resina composta na face incisal para restabelecimento da dimensão vertical e em cimento de ionômero de vidro restaurador convencional nas faces proximais. $\mathrm{O}$ paciente foi então reabilitado com prótese parcial removível superior mucossuportada e inferior dentomucossuportada (Figura 2 A e B). Foi realizado também clareamento dentário, em duas sessões, com peróxido de hidrogênio a 35\% dos dentes 33 ao 43 e prescrição de bochechos diários com fluoreto de sódio não acidulado a $0,05 \%$ por 30 dias.

Figura 2: Aspecto clínico final. A)Após tratamento restaurador e sepultamentos radiculares. B)Instalação de prótese total superior e prótese parcial removível inferior.
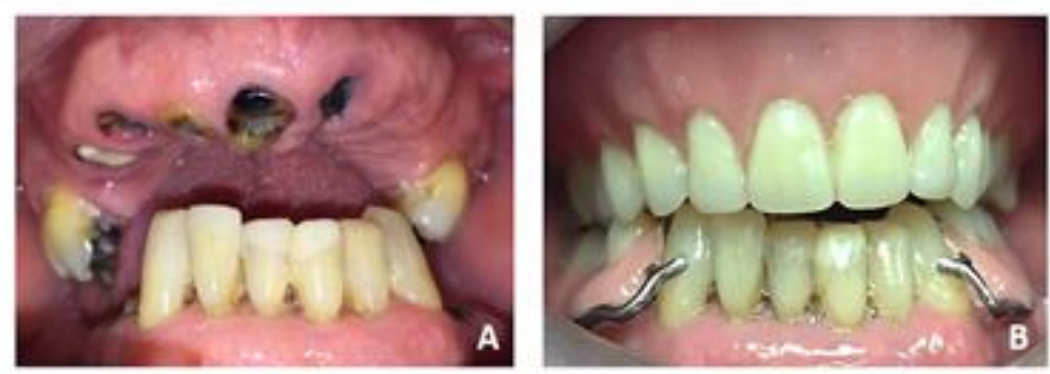

Fonte: Os Autores. 
Ao final do tratamento o paciente relatava conforto no uso das próteses e reestabelecimento da função mastigatória e estética dental. Assim, solicitou-se que o mesmo comparecesse a cada três meses para controle de biofilme e verificação da integridade das próteses e restaurações realizadas.

\section{Discussão}

A RT é, sem dúvidas, uma eficiente modalidade terapêutica para as neoplasias malignas na região de cabeça e pescoço. Porém, apesar de todos os avanços tecnológicos na área, a técnica ainda promove efeitos deletérios que muito frequentemente acometem a cavidade oral. Para o manejo, é imprescindível que os profissionais utilizem estratégias de prevenção e tratamento adequadas (Beech et al., 2014).

A xerostomia é definida como a sensação subjetiva de boca seca resultante ou não da secreção insuficiente de saliva (Tanasiewicz et al., 2016; Wiener et al., 2010). A diminuição do fluxo salivar, conhecida como hipossalivação, é uma das mais comuns alterações nos pacientes irradiados em cabeça e pescoço, ocasionando prejuízos em suas funções orais básicas, desconforto e maior susceptibilidade a infecções orais oportunistas e desenvolvimento de lesões cárie (Sood et al., 2014). Contudo, o padrão de desenvolvimento da cárie de radiação não é apenas dependente destas alterações salivares frente aos danos radioinduzidos nas glândulas, mas também dos efeitos diretos da radiação nas estruturas dentárias mineralizadas. Além disso, fatores como higiene oral deficiente, dieta cariogênica e alterações na microbiota oral também possuem papel importante no processo (Lieshout \& Bots, 2014; Ray-Chaudhuri et al., 2013).

O tratamento restaurador da cárie de radiação pode ser um desafio à seleção dos materiais dentários, pois encontra-se alteração do substrato dentário (esmalte e dentina) e ambiente oral hostil, os quais podem comprometer as propriedades de ligação de materiais adesivos. Assim, os cimentos de ionômeros de vidro provaram ser uma alternativa interessante para estes pacientes, além do uso de fluoretos, por reduzirem a atividade de cárie (Gupta et al., 2015; Lanzos et al., 2015; Silva et al., 2010). A associação da utilização do ionômero de vidro e uso de bochecho fluoretado teve por finalidade evitar a recidiva das lesões de cárie e oferecer condições restauradoras para a confecção das próteses.

Quando dentes com extensa destruição coronária estão presentes nestes pacientes irradiados, é bastante comum lançar mão da combinação de tratamento endodôntico e posterior sepultamento radicular, a fim de evitar ao máximo a realização de exodontias. Desta 
forma, previne-se também o surgimento de novas lesões de cáries de radiação e o risco de desenvolvimento de osteorradionecrose (Kielbassa et al., 1995; Lilly et al., 1998). Apesar de atualmente serem reportadas baixas taxas de incidência de ORN, esta parece mais prevalente em indivíduos do sexo masculino, de meia idade e com tumores em cavidade oral submetidos à RT conformacional-3D (Kuhnt et al., 2016).

A teoria mais aceita sobre o desenvolvimento da osteorradionecrose dos maxilares baseia-se no processo de fibrose radioinduzida resultante da ativação e alteração da atividade dos fibroblastos. Além do mais, esta pode ocorrer espontaneamente ou frente a traumas locais (cirurgias, próteses ou restaurações mal adaptadas) ou infecções orais (Lanzos et al., 2015). Acredita-se que a dose total de radiação também esteja fortemente relacionada à osteorradionecrose, porém a mandíbula parece ser mais susceptível que a maxila por apresentar suprimento sanguíneo limitado (Kuhnt et al., 2016). No presente caso clínico, o tratamento de escolha para o dente 27 foi a exodontia, já que a perda óssea avançada inviabilizava qualquer outro tratamento menos invasivo. Como possíveis medidas para a prevenção de osteorradionecrose baseadas em uma outra teoria que propõe infecção subsequente à trauma local em osso previamente exposto à radiação (Dhanda et al., 2016), prescreveu-se antibioticoterapia pós-operatória (Al-Bazie et al., 2016; de Menezes et al., 2014; Maxymiw et al., 1991) associada à terapia fotodinâmica antibacteriana (Campos et al., 2015). Esta terapia foi aplicada já que possui relatos bastante promissores na prevenção e tratamento da osteorradionecrose, além de não-invasiva. Ademais, é capaz de promover descontaminação local e importante efeito analgésico, contribuindo para um maior conforto no pós-operatório de forma segura e sem efeitos adversos (Campos et al., 2015).

Assim como observado no caso em questão, já é bem conhecido que a reabilitação oral dos pacientes com câncer de cabeça e pescoço impacta diretamente na qualidade de vida, envolvendo aspectos funcionais, psicológicos, sociais e físicos (Dholam et al., 2017).

\section{Considerações Finais}

O manejo odontológico de pacientes pós-irradiados em cabeça e pescoço deve ser cuidadosamente planejado e conduzido por uma equipe odontológica interdisciplinar, considerando as sérias consequências resultantes dos efeitos deletérios da RT na cavidade oral. Desta forma, pode-se contribuir diretamente na melhora da qualidade de vida deste grupo de pacientes. 


\section{Referências}

Al-Bazie, S., Bahatheq, M., Al-Ghazi, M., Al-Rajhi, N., \& Ramalingam, S. (2016). Antibiotic protocol for the prevention of osteoradionecrosis following dental extractions in irradiated head and neck cancer patients: A 10 years prospective study. Journal of Cancer Research and Therapeutics, 12(2), 565-570. https://doi.org/10.4103/0973-1482.159090

Beech, N., Robinson, S., Porceddu, S., \& Batstone, M. (2014). Dental management of patients irradiated for head and neck cancer. Australian Dental Journal, 59(1), 20-28. https://doi.org/10.1111/adj.12134

Comodo, G. V., Palma, L. F., Dos Santos, M. S., Seoanes, G. A., Gonnelli, F. A. S., Segreto, R. A., Segreto, H. R. C., \& Reimão, J. Q. (2020). Infecções orais oportunistas em pacientes submetidos à radioterapia para câncer de cabeça e pescoço: um estudo retrospectivo. Research, Society and Development, 9(3), 164932685. https://doi.org/10.33448/rsd-v9i3.2685

De Campos, L., Migliorati, C. A., \& Simões, A. (2015). Antimicrobial photodynamic therapy in cancer patients. In Lasers in Dentistry: Guide for Clinical Practice.

https://doi.org/10.1002/9781118987742.ch43

de Menezes, B. C., Noronha, V. R. A. S., Carvalho, A. L., Freire, A. R. S., \& Jham, B. C. (2014). Incidence of osteoradionecrosis following oral and maxillofacial surgery in irradiated head and neck cancer patients. Oral Surgery, 7(1), 12-17. https://doi.org/10.1111/ors.12054

Dhanda, J., Pasquier, D., Newman, L., \& Shaw, R. (2016). Current Concepts in Osteoradionecrosis after Head and Neck Radiotherapy. Clinical Oncology, 28(7), 459-466. https://doi.org/10.1016/j.clon.2016.03.002

Dholam, K. P., Dugad, J. A., \& Sadashiva, K. M. (2017). Impact of oral rehabilitation on patients with head and neck cancer: A study using the Liverpool Oral Rehabilitation Questionnaire and the Oral Health Impact Profile-14. The Journal of Prosthetic Dentistry, 117(4), 559-562. https://doi.org/10.1016/j.prosdent.2016.06.019

Gonnelli, F. A. S., Palma, L. F., Giordani, A. J., Deboni, A. L. S., Dias, R. S., Segreto, R. A., 
\& Segreto, H. R. C. (2016a). Low-Level Laser for Mitigation of Low Salivary Flow Rate in Head and Neck Cancer Patients Undergoing Radiochemotherapy: A Prospective Longitudinal Study. Photomedicine and Laser Surgery, 34(8), 326-330.

https://doi.org/10.1089/pho.2016.4104

Gonnelli, F. A. S., Palma, L. F., Giordani, A. J., Deboni, A. L. S., Dias, R. S., Segreto, R. A., \& Segreto, H. R. C. (2016b). Low-level laser therapy for the prevention of low salivary flow rate after radiotherapy and chemotherapy in patients with head and neck cancer. Radiologia Brasileira, 49(2), 86-91. https://doi.org/10.1590/0100-3984.2014.0144

Gupta, N., Pal, M., Rawat, S., Grewal, M., Garg, H., Chauhan, D., Ahlawat, P., Tandon, S., Khurana, R., Pahuja, A., Mayank, M., \& Devnani, B. (2015). Radiation-induced dental caries, prevention and treatment - A systematic review. National Journal of Maxillofacial Surgery, 6(2), 160. https://doi.org/10.4103/0975-5950.183870

Kielbassa, A. M., Attin, T., Schaller, H. G., \& Hellwig, E. (1995). Endodontic therapy in a postirradiated child: review of the literature and report of a case. Quintessence International, 26(6), 405-411.

Kielbassa, A. M., Hinkelbein, W., Hellwig, E., \& Meyer-Lückel, H. (2006). Radiation-related damage to dentition. The Lancet Oncology, 7(4), 326-335. https://doi.org/10.1016/S14702045(06)70658-1

Kuhnt, T., Stang, A., Wienke, A., Vordermark, D., Schweyen, R., \& Hey, J. (2016). Potential risk factors for jaw osteoradionecrosis after radiotherapy for head and neck cancer. Radiation Oncology, 11(1), 101. https://doi.org/10.1186/s13014-016-0679-6

Lanzos, I., Herrera, D., Lanzos, E., \& Sanz, M. (2015). A critical assessment of oral care protocols for patients under radiation therapy in the regional University Hospital Network of Madrid (Spain). Journal of Clinical and Experimental Dentistry, 7(5), e613-e621. https://doi.org/10.4317/jced.52557

Lieshout, H. F. J., \& Bots, C. P. (2014). The effect of radiotherapy on dental hard tissue-a systematic review. Clinical Oral Investigations, 18(1), 17-24. https://doi.org/10.1007/s00784- 
013-1034-z

Lilly, J. P., Cox, D., Arcuri, M., \& Krell, K. V. (1998). An evaluation of root canal treatment in patients who have received irradiation to the mandible and maxilla. Oral Surgery, Oral Medicine, Oral Pathology, Oral Radiology, and Endodontology, 86(2), 224-226.

https://doi.org/10.1016/S1079-2104(98)90129-9

Marta, G. N., Silva, V., de Andrade Carvalho, H., de Arruda, F. F., Hanna, S. A., Gadia, R., da Silva, J. L. F., Correa, S. F. M., Vita Abreu, C. E. C., \& Riera, R. (2014). Intensitymodulated radiation therapy for head and neck cancer: Systematic review and meta-analysis. Radiotherapy and Oncology, 110(1), 9-15. https://doi.org/10.1016/j.radonc.2013.11.010

Maxymiw, W. G., Wood, R. E., \& Liu, F. F. (1991). Postradiation dental extractions without hyperbaric oxygen. Oral Surgery, Oral Medicine, Oral Pathology, 72(3), 270-274. https://doi.org/10.1016/0030-4220(91)90212-U

Palma, L. F., Gonnelli, F. A. S., Marcucci, M., Dias, R. S., Giordani, A. J., Segreto, R. A., \& Segreto, H. R. C. (2017). Impact of low-level laser therapy on hyposalivation, salivary pH, and quality of life in head and neck cancer patients post-radiotherapy. Lasers in Medical Science, 32(4), 827-832. https://doi.org/10.1007/s10103-017-2180-3

Palma, L. F., Gonnelli, F. A. S., Marcucci, M., Giordani, A. J., Dias, R. S., Segreto, R. A., \& Segreto, H. R. C. (2018). A novel method to evaluate salivary flow rates of head and neck cancer patients after radiotherapy: a pilot study. Brazilian Journal of Otorhinolaryngology, 84(2), 227-231. https://doi.org/10.1016/j.bjorl.2017.03.004

Palma, L. F., Tateno, R. Y., Remondes, C. M., Marcucci, M., \& Cortes, A. R. G. (2020). Impact of radiotherapy on mandibular bone: A retrospective study of digital panoramic radiographs. Imaging Science in Dentistry, 50, 31-36.

https://doi.org/10.5624/isd.2020.50.1.31

Ray-Chaudhuri, A., Shah, K., \& Porter, R. J. (2013). The oral management of patients who have received radiotherapy to the head and neck region. British Dental Journal, 214(8), 387393. https://doi.org/10.1038/sj.bdj.2013.380 
Silva, A. R. S., Alves, F. A., Berger, S. B., Giannini, M., Goes, M. F., \& Lopes, M. A. (2010).

Radiation-related caries and early restoration failure in head and neck cancer patients. A polarized light microscopy and scanning electron microscopy study. Supportive Care in Cancer, 18(1), 83-87. https://doi.org/10.1007/s00520-009-0633-3

Sood, A. J., Fox, N. F., O’Connell, B. P., Lovelace, T. L., Nguyen, S. A., Sharma, A. K., Hornig, J. D., \& Day, T. A. (2014). Salivary gland transfer to prevent radiation-induced xerostomia: A systematic review and meta-analysis. Oral Oncology, 50(2), 77-83. https://doi.org/10.1016/j.oraloncology.2013.10.010

Tanasiewicz, M., Hildebrandt, T., \& Obersztyn, I. (2016). Xerostomia of Various Etiologies: A Review of the Literature. Advances in Clinical and Experimental Medicine, 25(1), 199206. https://doi.org/10.17219/acem/29375

Wiener, R. C., Wu, B., Crout, R., Wiener, M., Plassman, B., Kao, E., \& McNeil, D. (2010). Hyposalivation and xerostomia in dentate older adults R. J Am Dent Assoc, 141(3), 279-284. https://doi.org/10.14219/jada.archive.2010.0161

\section{Porcentagem de contribuição de cada autor no manuscrito}

Adriana Ferreira Granchelli - 20\%

Natália Silva Andrade - 20\%

Marina Gallottini - 10\%

Luana Campos - 10\%

Ricardo Yudi Tateno - 10\%

Luiz Felipe Palma - 10\%

Fabiana Martins - 20\% 